Supporting Information for

\title{
Dynamically Self-Reconfigurable Multifunctional All-Passive
}

\section{Metasurface}

Ratanak Phon, and Sungjoon Lim*

School of Electrical and Electronic Engineering, Chung-Ang University, Heukseok-Dong, Dongjak-Gu, Seoul, 06974, Republic of Korea.

*Corresponding Author: sungjoon@,cau.ac.kr 


\section{Supplementary Note 1: Proposed Power Modulated Unit Cell Geometrical Dimensions}

The proposed power modulated unit cell (see Figure S1A) was fabricated using printed circuit board (PCB) chemical etching process. The fabricated prototype was measured by placing it between two rectangular waveguides (430WCAS from Chengdu AINFO Inc) connected to an Agilent E5071C vector network analyzer (VNA), as shown in Figure S1B. Scattering parameters were measured with input RF power of $-20 \mathrm{dBm}$ and $10 \mathrm{dBm}$, correspond to low and high RF power modes, respectively.

A

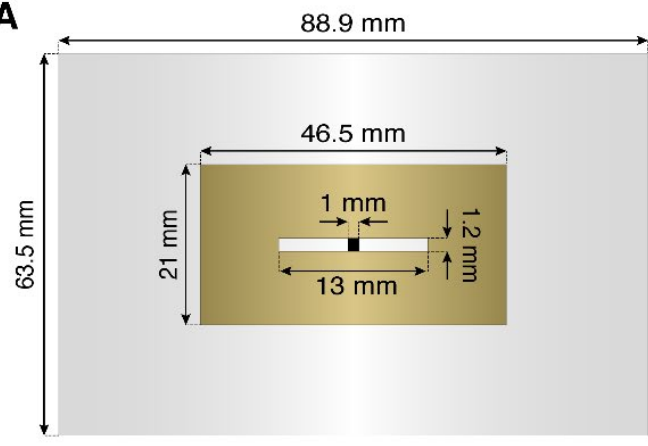

B

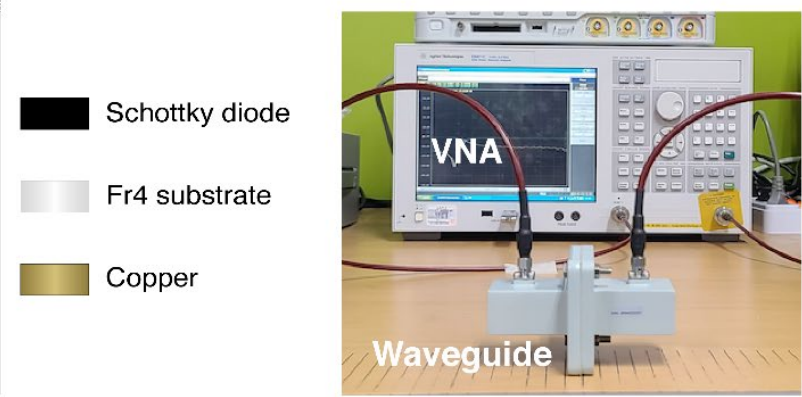

Figure S1. Power modulated unit cell. (A) Geometrical dimensions. (B) Measurement setup. 


\section{Supplementary Note 2: High Gain Antenna using Partially Reflective Surfaces}

The directivity of the patch antenna can be increased by studying multiple EM wave reflections between the ground plane and partially reflective surface (PRS). ${ }^{1,2}$ Figure S2A shows the schematic of the proposed metasurface antenna for the same case as in Figure 3A. Multiple reflections occur in the air cavity with reflected phases $\phi$ and $-\pi$ for the metasurface and ground plane, respectively. Gain, when $\theta=0$, can be expressed as

$$
G=\frac{1-\rho^{2}}{1+\rho^{2}-2 \rho \cos \left(\phi-\pi-\frac{4 \pi d}{\lambda}\right)}
$$

where $\rho$ and $\phi$ are reflected magnitude and phase of the metasurface, $d$ is the air space thickness, and $\lambda$ is wavelength. Thus, maximum gain can be obtained when

$$
\cos \left(\phi-\pi-\frac{4 \pi d}{\lambda}\right)=1
$$

or

$$
\phi-\pi=\frac{4 \pi d}{\lambda}-2 \pi N
$$

for $N=0, \pm 1, \pm 2, \ldots$ Therefore, boresight gain can be expressed as

$$
G=\frac{1+\rho}{1-\rho}
$$

or

$$
G_{\mathrm{dB}}=10 \log _{10}\left(\frac{1+\rho}{1-\rho}\right)
$$

Figure 3D shows the result obtained using MATLAB to numerically compute Equation (S3). 
A

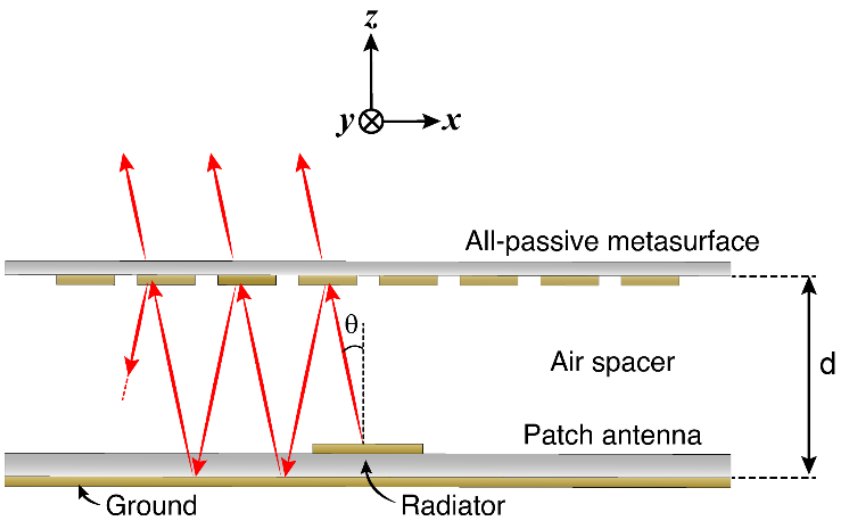

B

Equivalent circuit

of Metasurface

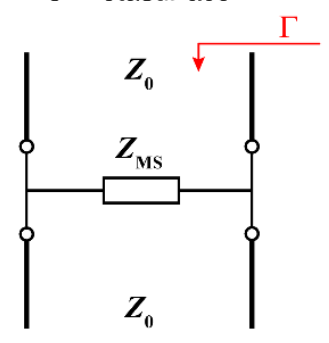

Figure S2. (A) Schematic illustration of the proposed structure which consists of patch antenna and metasurface separating by air spacer. (B) Equivalent circuit model of the metasurface. 


\section{Supplementary Note 3: Radar Cross Section Reduction}

Figure S3 shows the overall structure and equivalent circuit model. We assume there is no significant effect from the dielectric substrate due to its thickness, and patch antenna metallic patch due to its small size. Therefore, the antenna part can be modeled as a short termination of the transmission line, as shown in Figure S3B. The reflection coefficient can be expressed as

$$
R=\frac{Z_{\text {in }}-Z_{0}}{Z_{\text {in }}+Z_{0}}
$$

where $Z_{0}$ and $Z_{\text {in }}$ are free space and input impedance, respectively; and $Z_{\text {in }}$ can be determined from

$$
Z_{\text {in } 1}=j Z_{0} \tan (\beta d)
$$

and

$$
Z_{\text {in }}=Z_{\mathrm{MS}} \| Z_{\text {in } 1}
$$

where $Z_{\mathrm{MS}}$ are characteristic impedance of metasurface and $\beta=2 \pi / \lambda$ is a phase constant.

Absorption magnitude can be calculated from $A=1-|R|^{2}$ since the reflective metallic ground plane prevents transmission. The reflection coefficient $R$ should be minimized to obtain maximum absorptivity, i.e., $R=0$ or $Z_{\text {in }}=Z_{0}$. From Equation (S8), characteristic impedance of the metasurface $Z_{\mathrm{MS}}$ can be expressed as

$$
Z_{\mathrm{MS}}=\frac{Z_{0} Z_{\text {in } 1}}{Z_{\text {in } 1}-Z_{0}}
$$

or

$$
Z_{\mathrm{MS}}=Z_{0} \frac{j \tan \left(2 \pi \frac{d}{\lambda}\right)}{j \tan \left(2 \pi \frac{d}{\lambda}\right)-1}
$$


Finally, we can find $Z_{\mathrm{MS}}$ (which is consider to be $Z_{\mathrm{F} 2}$ for the receiving mode) as a function of distance per wavelength $(d / \lambda)$ from Equations (S10). Figure 3E was obtained using MATLAB to numerically compute $Z_{\mathrm{MS}}$ as a function of $(d / \lambda)$.

A

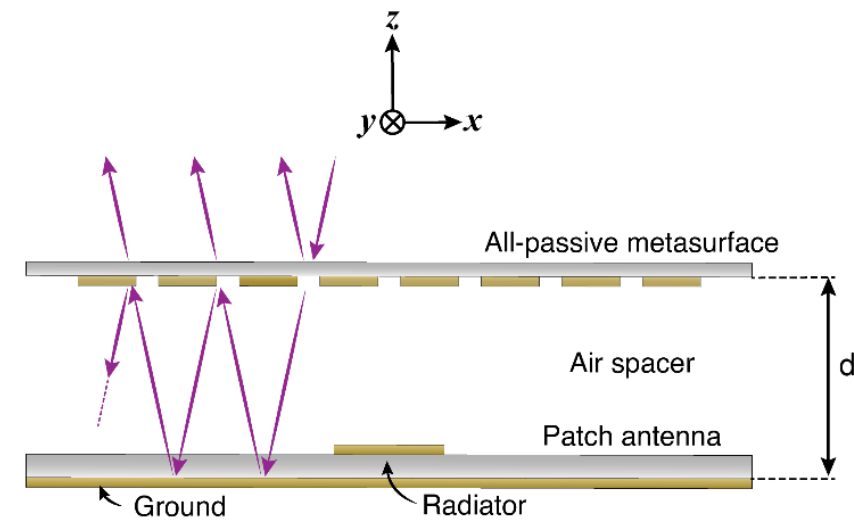

B

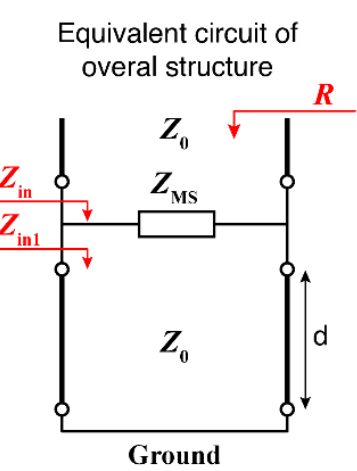

Figure S3. (A) Schematic illustration of the proposed structure comprising patch antenna and metasurface separated by an air space. (B) Corresponding equivalent circuit model. 


\section{Supplementary Note 4: Proposed Self-Reconfigurable Structure Geometry}

A

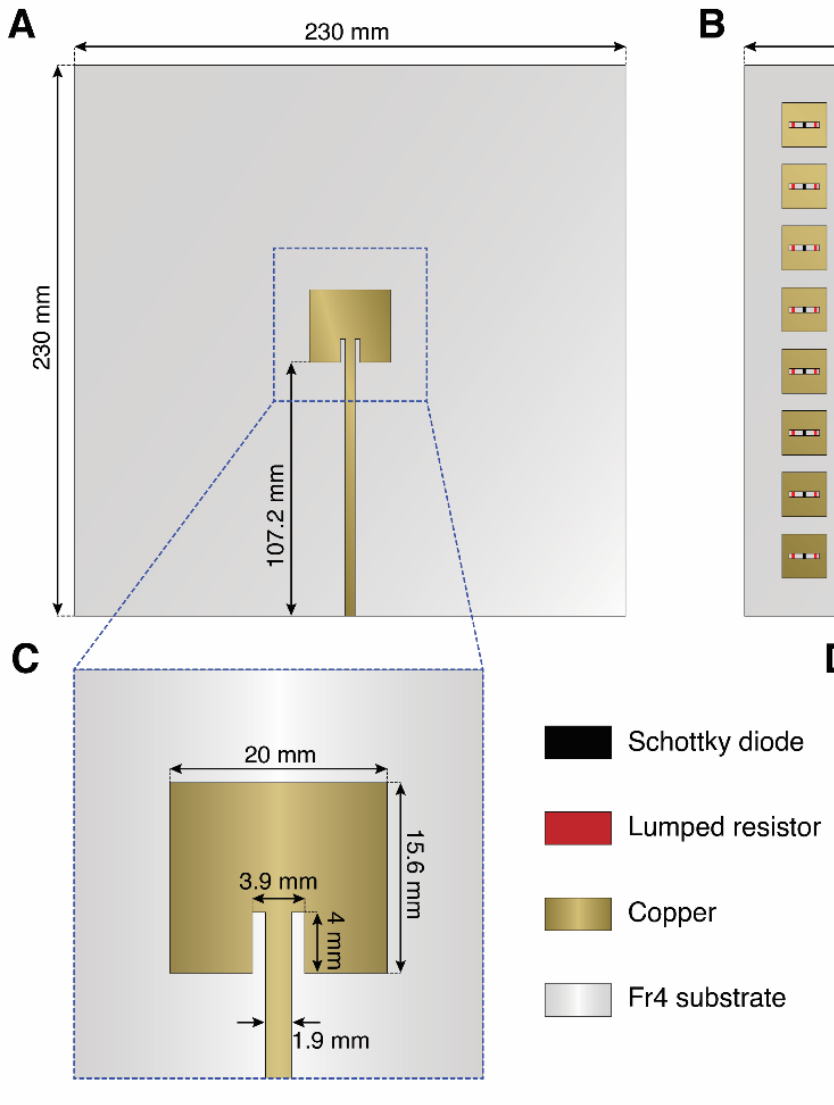

$230 \mathrm{~mm}$

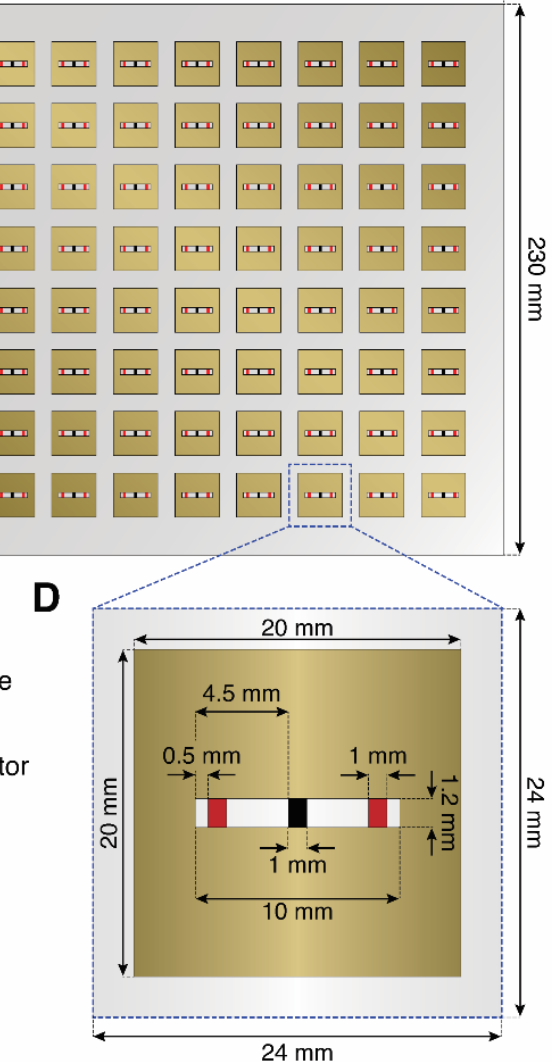

Figure S4. Geometry dimension and photograph of the fabricated prototype. (A) Patch antenna top view. (B) Proposed all-passive metasurface top view. (C) Patch antenna close view. (D) Proposed metasurface unit cell dimensions. 


\section{Supplementary Note 5: Theoretical Results of Proposed Self-Reconfigurable Structure}

Figure S5 shows theoretical predictions for realized gain and absorptivity to compare with the simulated results in Figure 5. These theoretical results were calculated using the simulated metasurface impedance in Figure 4D using Equations (S1) and (S6) from Supplementary Notes 2 and 3.
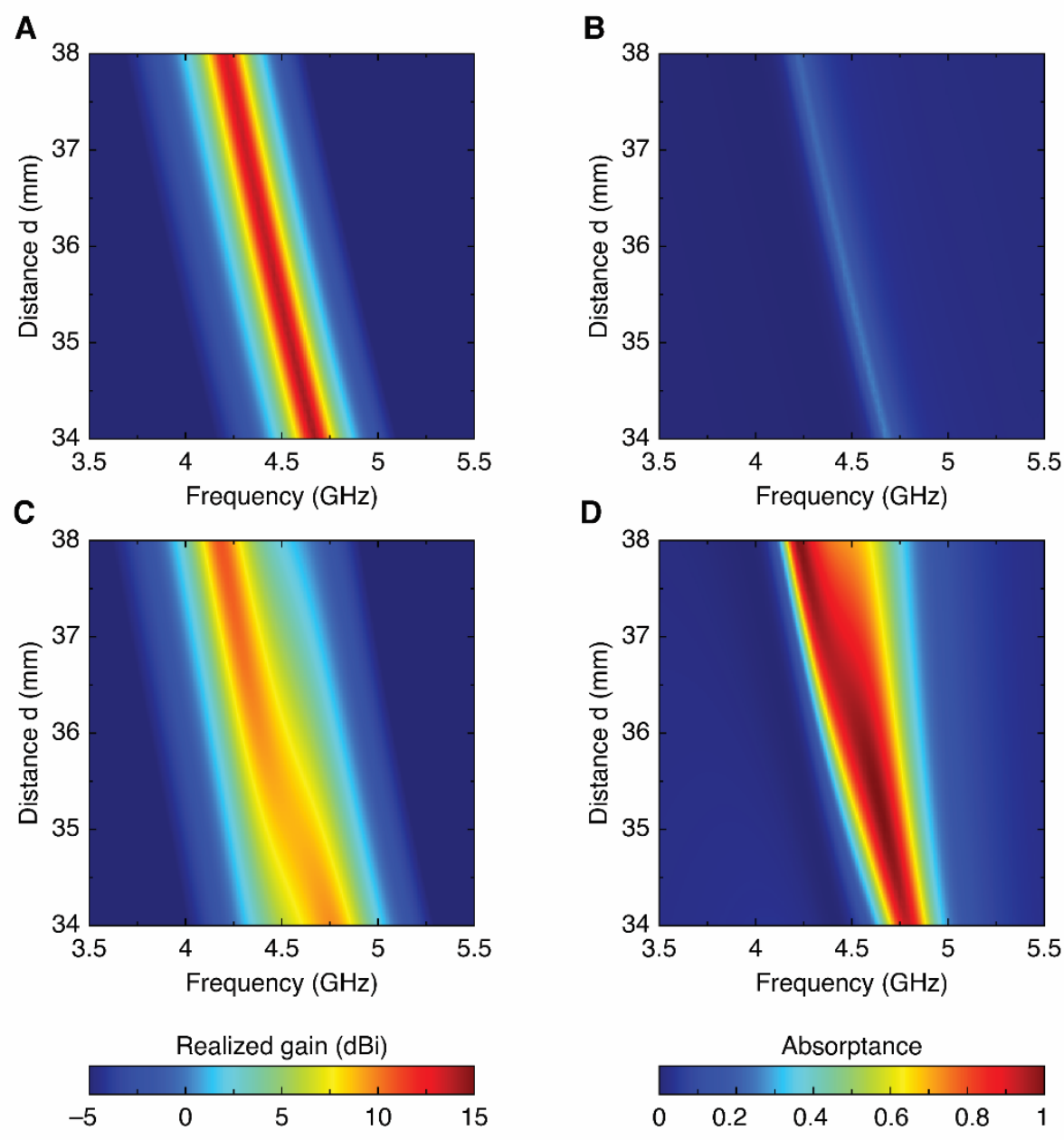

Figure S5. Theoretical EM responses of the proposed structure for different working states (ON and OFF states) and distances d (from 34 to $38 \mathrm{~mm}$ ). (A, B) Realized gain and absorptance in for ON state of the metasurface. (C, D) Realized gain and absorptance for OFF state. 


\section{References}

(1) Von Trentini, G. Partially Reflecting Sheet Arrays. IRE Trans. Antennas Propag. 1956, 4, 666-671.

(2) Feresidis, A. P.; Vardaxoglou, J. C. High Gain Planar Antenna Using Optimised Partially Reflective Surfaces. IEE Proc. Microwaves, Antennas Propag. 2001, 148, $345-350$. 